\title{
The line-of-sight distribution of water in SgrB2
}

\author{
Claudia Comito ${ }^{1}$, Peter Schilke ${ }^{1}$, Maryvonne Gerin ${ }^{2}$, Tom G. Phillips ${ }^{3}$, \\ Jonas Zmuidzinas ${ }^{3}$, and Darek C. Lis ${ }^{3}$ \\ 1 Max-Planck-Institut für Radioastronomie, Auf dem Hügel 69, D-53121 Bonn, \\ Germany ccomito@mpifr-bonn.mpg.de \\ 2 Laboratoire de Radioastronomie Millimétrique, Observatoire de Paris and Ecole \\ Normale Supérieure, 24 rue Lhomond, F-75231 Paris, CEDEX 05, France \\ 3 California Institute of Technology, Downs Laboratory of Physics 320-47, \\ Pasadena, CA 91125, USA
}

\section{$1 \mathrm{H}_{2} \mathrm{O}$ in $\mathrm{SgrB2}$}

The SgrB2 cloud is one of the most massive star forming regions in our Galaxy. It has many unique characteristics, among them an exceptional chemistry. Several species (FeO, [21]; $\mathrm{NH}_{2}$, [20]; $\mathrm{HF}$, [15]; $\mathrm{C}_{3}$, [7]) have, in spite of searches elsewhere, been detected only toward this source. One possible explanation for this enigmatic chemistry is the existence of a layer of hot gas, which is thought to be produced by a shock. Since this layer has the same velocity as the ambient gas in SgrB2, it has been proven difficult to assess its importance for the chemistry of many species, with a few exceptions. In particular its importance for the water chemistry has been a matter of recent debate $([3] ;[17])$. While its temperature and density are well known (cf. [9]), its column density and width have remained elusive. In this work (illustrated in detail in [5]) we have been able to determine its importance for water chemistry and give constraints for column density and spatial width, by modeling the $\mathrm{HDO}$ and $\mathrm{H}_{2}^{18} \mathrm{O}$ emission and absorption.

Water is known to be a fundamental ingredient of the interstellar medium. An ubiquitous tracer of shock-heated gas, it dramatically influences the chemistry in shocked regions (cf. [14]; [1]). In general, it acts as a a major coolant in star-forming clouds ([2]). Although direct observation of non-masing water lines from ground-based observatories is made extremely difficult by atmospheric absorption, $\mathrm{H}_{2} \mathrm{O}$ abundances can be estimated via observations of isotopomers, such as $\mathrm{H}_{2}^{18} \mathrm{O}$ (e.g. [19]; [10] and [11]; [6]), and, in recent years, by the availibility of satellites such as ISO (e.g. [4]) and notably SWAS (cf. [16]), but also by airborne observations of $\mathrm{H}_{2}^{18} \mathrm{O}$ (e.g. [23]). However, the deuterated counterpart of water, HDO, presents many features observable from ground in the $\mathrm{cm}, \mathrm{mm}$ and submm wavelength atmospheric windows (cf. [8]; [11] and [12]; [18]), and is also used as a tracer of water abundance under the assumption that both the deuterated and non-deuterated species be spatially coexistent, and that their abundance ratio remain constant throughout the studied region.

We report the detection of the $894-\mathrm{GHz}$ ground-state $1_{1,1}-0_{0,0}$ transition of HDO in absorption against the background continuum sources $\operatorname{SgrB2}(\mathrm{M})$ 

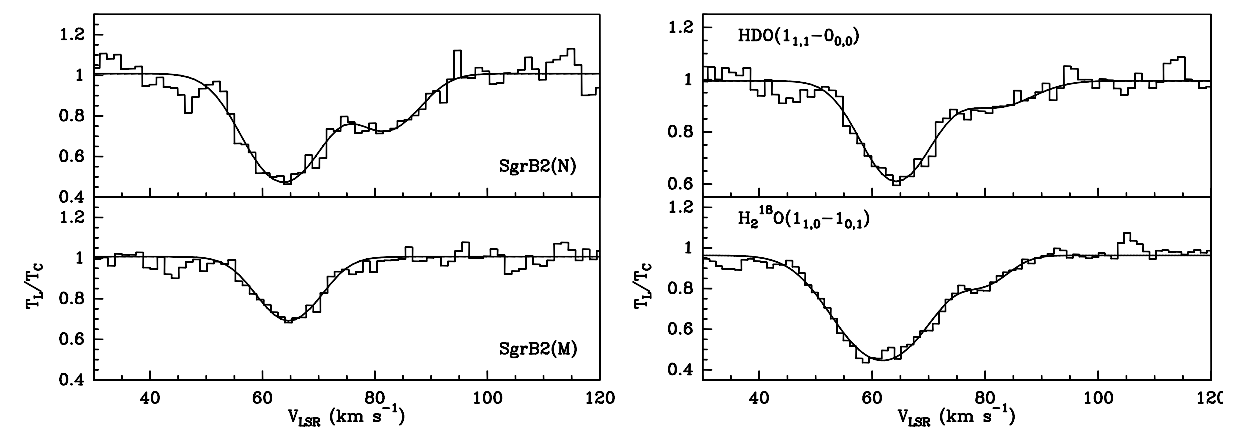

Fig. 1. Left panel: the $894-\mathrm{GHz}$ HDO $\left(1_{1,1}-0_{0,0}\right)$ transition, observed in absorption against the continuum emission of $\operatorname{SgrB2}(\mathrm{M})$ and of $\operatorname{SgrB} 2(\mathrm{~N})$. Right panel: comparison between the $894-\mathrm{GHz} \operatorname{HDO}\left(1_{1,1}-0_{0,0}\right)$ and the $547.7-\mathrm{GHz}$ o$\mathrm{H}_{2}^{18} \mathrm{O}\left(1_{1,0}-1_{0,1}\right)$ absorption features observed towards SgrB2. The $y$-scale gives the line-to-continuum $\left(\mathrm{T}_{\mathrm{L}} / \mathrm{T}_{\mathrm{C}}\right)$ ratio.

and SgrB2(N). The radial velocity of the observed HDO features suggests that the absorption is directly connected to the SgrB2 complex. The fact that, i), we observed a ground-state transition, and ii), we observed it in absorption, allowed us to estimate, indipendently of assumption on the physical state of the gas, the HDO column density, which we found to be of $1.2 \times 10^{13} \mathrm{~cm}^{-2}$ towards $\operatorname{SgrB} 2(\mathrm{M})$, and of $2.4 \times 10^{13}$ and $1.2 \times 10^{13} \mathrm{~cm}^{-2}$ towards the 65 - and the $81-\mathrm{km} \mathrm{s}^{-1}$ velocity components of $\mathrm{SgrB} 2(\mathrm{~N})$ respectively. Moreover, using the $\mathrm{H}_{2}^{18} \mathrm{O}$ SWAS data $([16])$, we were able to estimate a lower limit of $5 \times 10^{-4}$ for the $[\mathrm{HDO}] /\left[\mathrm{H}_{2} \mathrm{O}\right]$ ratio towards the $\mathrm{SgrB} 2$ complex.

The above mentioned results hold under the assumption that all the absorbing water lies in the warm envelope. [3] propose that $\mathrm{H}_{2} \mathrm{O}$ might instead be located in the hot gas layer that surrounds the whole SgrB2 complex, implying that gas-phase water in the warm envelope does not contribute at all to the observed absorption features. In fact, while this hypothesis is reasonable for $\mathrm{H}_{2} \mathrm{O}$ (the gas-phase production of water is triggered by high temperatures), it would not be applicable to HDO (the same high temperatures inhibit its enhancement). We therefore think it possible that the gas observed in $\mathrm{HDO}$ and that seen in $\mathrm{H}_{2} \mathrm{O}$ absorption be located in distinct regions.

\section{Modeling the distribution of water}

SgrB2 is often adopted as a template region for the study of sites of massive star formation. For this purpose, it is important to evaluate to what extent the warm envelope and the hot layer separately contribute to the observed HDO 
and $\mathrm{H}_{2} \mathrm{O}$ absorption. In particular, in order to better understand the nature of the hot layer and possibly the mechanism that created it, it is vital to learn more about its chemical and physical characteristics, and especially about its water content. A precise determination of the column density of water would set a tight constraint on the physical models that attempt to identify the heating mechanism responsible for the high gas temperatures. Unfortunately, since both gas components show roughly the same radial velocity, it is not possible to separate, by purely observational means, the ground-state water absorption produced in the warm envelope from that produced in the hot layer. However, a number of $\mathrm{HDO}$ and $\mathrm{H}_{2}^{18} \mathrm{O}$ transitions have been observed towards the SgrB2 cores with a variety of instruments.

Our 893-GHz HDO data, together with the published $\mathrm{HDO}$ and $\mathrm{H}_{2}^{18} \mathrm{O}$ observations carried out at $\mathrm{mm}$ and submm wavelengths, provide strong observational constraints and enable us to model, in a self-consistent manner, the $\mathrm{HDO}$ and $\mathrm{H}_{2} \mathrm{O}$ abundance in all three components of the SgrB2 cloud. In detail:

a) the observed emission lines of $\mathrm{p}-\mathrm{H}_{2}^{18} \mathrm{O}$ at $203 \mathrm{GHz}(\mathrm{SgrB} 2(\mathrm{~N}),[6])$, and of HDO at 143, 226, $241 \mathrm{GHz}(\mathrm{SgrB} 2(\mathrm{M})$ and $(\mathrm{N}),[11])$ constrain the HDO and water abundance and the $\mathrm{H}_{2}$ density in the hot-core-type components;

b) the 893-GHz HDO absorption feature observed towards SgrB2(M) and N, together with the $[\mathrm{HDO}] /\left[\mathrm{H}_{2} \mathrm{O}\right]$ ratio estimated at point $a$ ), help us set the $\mathrm{HDO}$ and $\mathrm{H}_{2} \mathrm{O}$ abundance in the warm envelope;

c) finally, having estimated the column density of water in the warm envelope, it is possible to assess whether or not the quantity of non-deuterated water in the warm envelope can be responsible for the $\mathrm{H}_{2}^{18} \mathrm{O}$ absorption observed at $548 \mathrm{GHz}([16],[23])$.

We use the radiative transfer code described by [24], with a few modifications (see [5] for details), to reproduce the intensities observed for the features. The model consists of two spherically symmetric components representing the hot core and the warm envelope (see $\S 1$ ). Most of the spectral line data are reproduced within errors of $\sim 30 \%$, with two exceptions:

1) The 226-GHz HDO emission line observed towards $\mathrm{SgrB} 2(\mathrm{M})$ is a factor of 2 weaker, and the $143-\mathrm{GHz} \mathrm{HDO}$ line in $\mathrm{SgrB} 2(\mathrm{~N})$ is 4 times stronger than predicted by the model. Such discrepancies are likely to be due to the intrinsic chemical differences between the two hot cores (cf. [13] and references therein), which has not been taken into account in our model.

2) Most interestingly, the predicted ground-state $\mathrm{o}-\mathrm{H}_{2}^{18} \mathrm{O}$ absorption at 548 $\mathrm{GHz}$ is much shallower than observed ([16], cf. Fig. 1): the abundance of non-deuterated water in the warm envelope is not sufficient to produce the absorption feature observed at $548 \mathrm{GHz}$.

This is a very important result: our predictions confirm, at least from a qualitative point of view, the hypothesis of [3] that the most important contribution to the observed $\mathrm{H}_{2}^{18} \mathrm{O}$ absorption come from the foreground hot gas 
layer. Having predicted the contribution of the warm envelope to the $\mathrm{H}_{2}^{18} \mathrm{O}$ absorption, it is possible to estimate the spatial width and $\mathrm{H}_{2} \mathrm{O}$ abundance necessary for the hot layer to produce the observed feature, given the observational constraints mentioned in section $\S 1$. We use the radiative transport equation to calculate the total intensity emerging from the hot layer, given the background emission produced by hot cores and warm envelope. The data are best reproduced when the $\mathrm{o}-\mathrm{H}_{2}^{18} \mathrm{O}$ column density in the hot layer is $\sim 10^{14} \mathrm{~cm}^{-2}$. Assuming a $\left[{ }^{16} \mathrm{O}\right] /\left[{ }^{18} \mathrm{O}\right]$ ratio of $261 \pm 20([22])$ and an ortho/para ratio of 3 , we can estimate the $\mathrm{H}_{2} \mathrm{O}$ column density in the hot gas component to be of order $\sim 3.5 \times 10^{16} \mathrm{~cm}^{-2}$. If all the atomic oxygen not locked in $\mathrm{CO}$ is bound in gas-phase water, then the $\mathrm{H}_{2} \mathrm{O}$ abundance in this region is about $5 \times 10^{-4} \mathrm{~cm}^{-3}$, and the spatial width of the hot layer is only $0.02 \mathrm{pc}$.

\section{References}

1. Bergin, E.A., Melnick, G.J., Neufeld, D.A. 1998, ApJ, 499, 777

2. Ceccarelli, C., Hollenbach, D.J., Tielens, A.G.G.M. 1996, ApJ, 471, 400

3. Ceccarelli, C., Baluteau, J.-P., Walmsley, C.M., et al. 2002, A\&A, 383, 603

4. Cernicharo, J., Lim, T., Cox, P. et al. 1997, A\&A, 323, 25

5. Comito, C., Schilke, P., Gerin, M., et al. 2003, A\&A, 402, 635

6. Gensheimer, P.D., Mauersberger, R., Wilson, T.L. 1996, A\&A, 314, 281

7. Giesen, T.F., Van Orden, A.O., Cruzan, J.D., et al. 2001, ApJ, 551, 181

8. Henkel, C., Mauersberger, R., Wilson, T.L., et al. 1987, A\&A, 182, 299

9. Hüttemeister, S., Wilson, T.L., Mauersberger, R., et al. 1995, A\&A, 294, 667

10. Jacq, T., Jewell, P.R., Henkel, C., et al. 1988, A\&A, 199, 5

11. Jacq, T., Walmsley, C.M., Henkel, C., et al. 1990, A\&A, 228, 447

12. Jacq, T., Baudry, A., Walmsley, C.M., et al. 1999, A\&A, 347, 957

13. Miao, Y., Snyder, L.E. 1997, ApJ, 480, L67

14. Neufeld, D.A., Melnick, G.J. 1987, ApJ, 322, 266

15. Neufeld, D. A., Zmuidzinas, J., Schilke, P., et al. 1997 ApJ, 488, 141

16. Neufeld, D.A., Ashby, M.L.N., Bergin, N.R. et al. 2000, ApJ, 539, 111

17. Neufeld, D. A., Bergin, E. A., Melnick, G. J., et al. 2003, ApJ, 590, 882

18. Pardo, J. R., Cernicharo, J., Herpin, F., et al. 2001, ApJ, 562, 799

19. Phillips, T.G., Scoville, N.Z., Kwan, J., et al. 1978, ApJ, 222, 59

20. van Dishoeck, E.F., Jansen, D.J., Schilke, P., et al. 1993, 1993 ApJ, 416, 83

21. Walmsley, C.M., Bachiller, R., Pineau des Forêts, G., et al. 2002 ApJ, 566, 109

22. Whiteoak, J.B., Gardner, F.F. 1981, MNRAS, 197, 39

23. Zmuidzinas, J., Blake, G.A., Carlstrom, J., et al. 1995b, Proceedings of the Airborne Astronomy Symposium on the Galactic Ecosystem: From Gas to Stars to Dust, ed. M.R. Haas, J.A. Davidson and E.F. Erickson, San Francisco: ASP 24. Zmuidzinas, J., Blake, G.A., Carlstrom, J., et al. 1995, ApJ, 447, L125 